\title{
Conhecimento de acadêmicos da área da saúde sobre a vacinação antitetânica
}

\section{Health care academics knowledge on tetanus vaccination}

\author{
Manoelito Ferreira Silva Junior ${ }^{1}$, Rahyza Inácio Freire de Assis², Cláudia Lobelli Rangel Gomes³, \\ Paula Vitali Miclos ${ }^{4}$, Hedilberto Araújo de Sousa ${ }^{5}$, Maria José Gomes ${ }^{5}$ \\ ${ }^{1}$ Programa de Pós-graduação em Odontologia, Departamento de Odontologia Social, Faculdade de Odontologia de \\ Piracicaba (FOP), Universidade Estadual de Campinas (UNICAMP) - Piracicaba (SP), Brasil. \\ ${ }^{2}$ Programa de Pós-graduação em Clínica Odontológica, Faculdade de Odontologia de Piracicaba (FOP), \\ UNICAMP - Piracicaba (SP), Brasil. \\ ${ }^{3}$ Programa de Pós-graduação em Odontologia, Departamento de Odontologia Infantil, Faculdade de Odontologia \\ de Piracicaba (FOP), UNICAMP - Piracicaba (SP), Brasil. \\ ${ }^{4}$ Programa de Pós-graduação em Auditoria, Faculdade Israelita de Ciências da Saúde Albert Einstein - São \\ Paulo (SP), Brasil. \\ ${ }^{5}$ Programa de Pós-graduação Profissional em Clínica Odontológica, Universidade Federal do Espírito Santo \\ (UFES) - Vitória (ES), Brasil.
}

\section{Resumo}

Introdução: O tétano é uma doença infecciosa, não contagiosa e ainda considerada um problema de saúde pública no Brasil. Objetivo: Investigar a cobertura vacinal e o conhecimento de acadêmicos dos cursos de Medicina, Odontologia e Enfermagem da Universidade Federal do Espírito Santo (UFES) sobre a vacina antitetânica. Metodologia: $\mathrm{O}$ estudo foi descritivo transversal com abordagem quantitativa. A determinação da amostra utilizou-se de cálculo amostral, determinado em 296 acadêmicos. Os critérios de inclusão foram: estar regulamente matriculado nos cursos de Odontologia, Medicina ou Enfermagem no semestre letivo de 2010/2, possuir mais de 18 anos e assinar o Termo de Consentimento Livre e Esclarecido. Resultados: Houve participação de 298 acadêmicos. Embora a maioria dos acadêmicos da área da saúde, 74 (91,36\%) do curso de Enfermagem, 133 (97,79\%) de Medicina e 74 (91,36\%) de Odontologia tenham o conhecimento de que o tétano é uma doença imunoprevenível, nem todos estão vacinados. Detectou-se que 74 (91,36\%) acadêmicos de Enfermagem, 115 (84,56\%) de Medicina e 62 (76,55\%) de Odontologia são vacinados contra o tétano. Conclusão: O conhecimento dos acadêmicos sobre a vacina antitetânica foi satisfatório, no entanto, nem todos estavam vacinados.

Palavras-chave: tétano; riscos ocupacionais; imunização; vacinação.

\begin{abstract}
Introduction: Tetanus is an infectious, non-contagious disease still considered a public health problem in Brazil. Aim: To investigate the vaccination coverage and knowledge of Medicine, Dentistry and Nursing scholars, from the Federal University of Espírito Santo (UFES), on the tetanus vaccine. Methodology: Cross-sectional study with a quantitative approach. The determination of the sample was used for sample size calculation and consisted of 296 academics. Inclusion criteria were: being regularly
\end{abstract}

\footnotetext{
Autor para correspondência: Manoelito Ferreira Silva Junior - Departamento de Odontologia Social, Faculdade de Odontologia de Piracicaba (FOP), Universidade Estadual de Campinas (UNICAMP) - Av. Limeira, 901 - Vila Rezende - CEP: 13414-903 - Piracicaba (SP), Brasil - E-mail: manoelito_fsjunior@hotmail.com Recebido em: 12 de novembro de 2016
} Aceito para publicação em: 04 de setembro de 2017 
enrolled in the courses of Dentistry, Medicine and Nursing in semester 2010/2, age 18 and older and signing the consent form. Results: There were 298 students who participated. Although most scholars in healthcare, 74 (91.36\%) nursing students, 133 (97.79\%) Medicine and 74 (91.36\%) Dentistry, have the knowledge that tetanus is an immune-preventable disease, not everyone is vaccinated. We found that $74(91.36 \%)$ nursing students, $115(84.56 \%)$ of Medicine and $62(76.55 \%)$ of Dentistry were vaccinated against tetanus. Conclusion: Academic knowledge about the tetanus vaccine was satisfactory; however, not all scholars were vaccinated.

Keywords: tetanus; occupational risks; immunization; vaccination.

\section{Introdução}

O tétano é uma doença infecciosa não contagiosa causada por um bacilo denominado Clostridium tetani, considerado de alta letalidade (VERONESI; FOCACCIA, 2015). O tétano é uma importante causa de morbimortalidade, principalmente nos países em desenvolvimento (VIERTEL et al., 2005).

A sua incidência está associada a piores condições socioeconômicas, baixa cobertura vacinal (OLIVEIRA, 2008), riscos ambientais e comportamentais (VIERTEL et al., 2005), principalmente em atividades laborais ou de lazer entre indivíduos não imunizados expostos a locais de risco para o agente etiológico (GOMERI; GAGLIANI, 2011).

No Brasil, vem se observando uma redução significativa do número de casos de tétano a cada ano (VIERTEL et al., 2005; FEIJÃO et al., 2007; BRASIL, 2009; GOUVEIA et al., 2009). Em 1982, foram confirmados 2.226 casos de tétano no Brasil, em 1992 ocorreram 1.312 novos casos, com uma redução de 58\%. Em 2002 ocorreram 617 casos, e em 2006 foram 415 novos casos. Portanto, desde a década de 1980 até o ano de 2006, houve uma redução de mais de $80 \%$ em todo o país (BRASIL, 2009). A partir dos anos 1990, a zona urbana passou a responder pelo maior número de casos da doença no Brasil, e as profissões mais acometidas são agricultores, pedreiros e trabalhadores de serviços de limpeza (FEIJÃO et al., 2007; OLIVEIRA, 2008) - perfil epidemiológico semelhante a países desenvolvidos (MORAES; PEDROSO, 2000).

Apesar de a imunização ativa ser considerada uma medida segura e efetiva para a profilaxia do tétano, com vacinação gratuita nos serviços públicos de saúde, os níveis de desenvolvimento humano e de cobertura vacinal são ainda considerados inadequados (OLIVEIRA et al. 2012). Embora exista o investimento público na prevenção da doença, ainda há um grande número de pessoas não imunizadas em todo o território nacional (FEIJÃO et al., 2007; GOMERI; GAGLIANI, 2011), inclusive profissionais da área da saúde. Isto revela a falta do autocuidado na formação acadêmica dos próprios profissionais de saúde, em que o foco é o conhecimento aplicado aos pacientes, sem comprometer-se como parte integrante deste sistema (PINHEIRO; ZEITOUNE, 2008).

Os profissionais de saúde estão diariamente expostos aos acidentes de trabalho, às doenças profissionais e às doenças do trabalho, inerentes ao desempenho de suas atividades clínicas (FRANÇA, 1999), por isso, os alunos da área da saúde classificam-se como grupo de risco às doenças infecciosas, incluindo-se o tétano. Nesse contexto, tornam-se necessárias ações de promoção de saúde e proteção específica nas áreas de maior vulnerabilidade (GOMERI; GAGLIANI, 2011). O profissional de saúde deve compreender que, além do uso constante dos equipamentos individuais de proteção (EPI) (GARCIA; FACCHINI, 2008), a imunização é o meio mais seguro para a proteção contra o tétano.

O objetivo do estudo foi investigar a cobertura vacinal e o conhecimento sobre a vacina antitetânica de acadêmicos dos cursos de Medicina, Odontologia e Enfermagem do Centro de Ciências da Saúde da Universidade Federal do Espírito Santo.

\section{Material e Métodos}

$\mathrm{O}$ presente trabalho caracteriza-se por um estudo descritivo transversal com abordagem quantitativo. A população do estudo foi composta por alunos dos cursos de Odontologia, Enfermagem e Medicina da Universidade Federal do Espírito Santo matriculados no semestre 2010/2.

O cálculo amostral foi determinado pela fórmula de Barnett (1982), com prevalência de 50\%, nível de confiança de $95 \%$ e margem de erro de $5 \%$, estando determinada em 296 alunos, subdivididos 
proporcionalmente pelo número de alunos ingressos em cada curso, sendo determinado em 12 acadêmicos de cada turma de Medicina, 9 alunos de cada turma de Odontologia e Enfermagem.

E os critérios de inclusão eram: acadêmicos cursando qualquer período dos cursos de Medicina, Odontologia e Enfermagem devidamente matriculados no semestre letivo de 2010/2 da Universidade Federal do Espírito Santo, ser maior de 18 anos, ter sido sorteado no estudo randomizado e ter assinado o Termo de Consentimento Livre e Esclarecido.

Os critérios de exclusão foram: o aluno não estar matriculado no semestre letivo de 2010/2 ou não aceitar assinar o Termo de Consentimento Livre e Esclarecido.

Os dados foram coletados a partir da aplicação de um questionário validado pelo estudo de Silva-Junior et al. (2013), que contempla perguntas sobre a vacinação, o agente etiológico, quantidade de doses para imunização, necessidade de reforço, dentre outros aspectos. Os dados foram tabulados em frequências absolutas e relativas no programa Microsoft Excel 2010. O estudo foi previamente aprovado pelo Comitê de Ética em Pesquisa da Ufes $\left(n^{\circ} 052 / 10\right)$.

\section{Resultados}

A amostra foi constituída por 298 acadêmicos dos cursos de Enfermagem, Medicina e Odontologia da Ufes (Tabela 1). Houve perda de oito voluntários de Medicina, devido a dados incompletos no questionário, o que prejudicou a amostra esperada. A maioria dos participantes era do sexo feminino (60,81\%), com média relativa de 22 anos.

Quando os alunos foram questionados se têm/tiveram tétano, $80(98,76 \%)$ alunos de Odontologia, 78 (96,30\%) alunos de Enfermagem e $136(100,00 \%)$ alunos de Medicina disseram não ter tido a doença. Os demais acadêmicos responderam não saber. E nenhum respondeu ter apresentado a doença em alguma fase da vida.

A Tabela 2 apresenta os dados referentes à realização de vacinação e ao conhecimento sobre a natureza imunoprevenível, infecciosa e transmissível do tétano, além do conhecimento sobre seu agente etiológico.

Quando perguntados sobre o conhecimento das manifestações clínicas do tétano, 129 (96,27\%) graduandos de Medicina, 64 (74,01\%) de Enfermagem e $39(48,15 \%)$ de Odontologia afirmaram saber as manifestações clínica da doença.
A Tabela 3 expressa os resultados da quantidade de doses que os acadêmicos achavam necessárias para completa cobertura vacinal para o tétano.

Questionamos se a vacina de tétano precisa de reforço, na Medicina 116 (86,57\%) disseram que necessita de reforço, $11(8,20 \%)$ marcaram "Não sei" como resposta. Em Enfermagem, $68(83,95 \%)$ afirmaram a necessidade de reforço, $13(16,05 \%)$ marcaram a opção "Não" e "Não sei". Na Odontologia, $55(67,90 \%)$ marcaram a opção "Sim", 20 (24,69\%) disseram não saber sobre a necessidade de reforço para o tétano.

\section{Discussão}

O tétano é uma doença que pode atingir igualmente os sexos e acometer as diversas faixas etárias, sendo mais comum nos países em desenvolvimento. O risco de aquisição do tétano acidental em pessoas não imunizadas ou imunizadas inadequadamente existe em qualquer lugar, uma vez que o agente etiológico Clostridium tetani tem distribuição universal (GOMERI; GAGLIANI, 2011).

Embora a maioria dos acadêmicos participantes desta pesquisa teve conhecimento sobre a doença tétano ser imunoprevenível, a Tabela 2 mostra que nem todos foram vacinados contra a doença. Esta questão é importante, uma vez que, mesmo tendo o conhecimento sobre a vacina antitetânica e se tratando de profissionais de saúde, ainda assim não são vacinados contra a doença (GOUVEIA et al., 2009). Os anticorpos protetores são induzidos exclusivamente pela aplicação da vacina antitetânica, uma vez que a neurotoxina, em razão de atuar em quantidades extremamente reduzidas, é capaz de

Tabela 1. Caracterização da amostra de acadêmicos da área da saúde da Universidade Federal do Espírito Santo que responderam sobre vacinação antitetânica. Vitória, Espírito Santo, 2010.

\begin{tabular}{lcc}
\hline \multicolumn{1}{c}{ Características } & $\mathbf{n}$ & $\mathbf{\%}$ \\
\hline Sexo & & \\
$\quad$ Masculino & 116 & $38,9 \%$ \\
$\quad$ Feminino & 182 & $61,1 \%$ \\
Idade & & \\
$\quad 18-23$ anos & 204 & $68,5 \%$ \\
$24-30$ anos & 94 & $31,5 \%$ \\
Curso & & \\
$\quad$ Medicina & 136 & $45,70 \%$ \\
$\quad$ Enfermagem & 81 & $27,15 \%$ \\
$\quad$ Odontologia & 81 & $27,15 \%$ \\
\hline
\end{tabular}


Tabela 2. Conhecimento dos acadêmicos da área da saúde acerca da doença tétano por curso*. Vitória, Espírito Santo, 2010

\begin{tabular}{|c|c|c|c|c|c|c|c|c|}
\hline \multirow{2}{*}{ Variável } & \multicolumn{2}{|c|}{ Enfermagem } & \multicolumn{2}{|c|}{ Medicina } & \multicolumn{2}{|c|}{ Odontologia } & \multicolumn{2}{|c|}{ Total } \\
\hline & $\mathbf{n}$ & $\%$ & n & $\%$ & $\mathbf{n}$ & $\%$ & $\mathbf{n}$ & $\%$ \\
\hline É uma doença infecciosa? & 54 & 66,67 & 112 & 82,35 & 42 & 51,85 & 208 & 69,80 \\
\hline Conhece o agente etiológico? & 52 & 64,20 & 133 & 97,79 & 45 & 55,56 & 230 & 77,18 \\
\hline É uma doença transmissível? & 17 & 20,99 & 31 & 22,79 & 29 & 35,80 & 77 & 25,84 \\
\hline É uma doença imunoprevenível? & 74 & 91,36 & 133 & 97,79 & 74 & 91,36 & 281 & 94,30 \\
\hline É vacinado? & 74 & 91,36 & 115 & 84,56 & 62 & 76,54 & 251 & 84,23 \\
\hline
\end{tabular}

*Foram contabilizados apenas os participantes que responderam "Sim". O total não contabilizado nesta tabela refere-se às opções "Não" e "Não sei”.

Tabela 3. Informação dos acadêmicos da área da saúde sobre quantidade de doses para maior cobertura vacinal do tétano. Vitória, Espírito Santo, 2010.

\begin{tabular}{|c|c|c|c|c|c|c|c|c|c|c|}
\hline \multirow{2}{*}{ Curso } & \multicolumn{2}{|c|}{3 doses } & \multicolumn{2}{|c|}{2 doses } & \multicolumn{2}{|c|}{1 dose } & \multicolumn{2}{|c|}{ Não sabe } & \multicolumn{2}{|c|}{ Total } \\
\hline & n & $\%$ & $\mathbf{n}$ & $\%$ & $\mathbf{n}$ & $\%$ & $\mathbf{n}$ & $\%$ & n & $\%$ \\
\hline Medicina & 27 & 19,85 & 45 & 33,09 & 36 & 26,47 & 28 & 20,59 & 136 & 100,00 \\
\hline Odontologia & 32 & 39,50 & 22 & 27,16 & 25 & 30,86 & 2 & 2,48 & 81 & 100,00 \\
\hline Enfermagem & 26 & 32,10 & 21 & 25,93 & 11 & 13,58 & 23 & 28,39 & 81 & 100,00 \\
\hline Total & 85 & 28,52 & 88 & 29,53 & 72 & 24,16 & 53 & 17,79 & 298 & 100,00 \\
\hline
\end{tabular}

produzir a doença, mas não a imunidade (GOMERI; GAGLIANI, 2011).

No presente estudo, os alunos possuíam conhecimento sobre o caráter infeccioso do tétano, e houve uma disparidade entre o conhecimento dos acadêmicos do curso de Medicina em relação aos alunos de Enfermagem e Odontologia. Quanto à natureza de transmissibilidade do tétano, a maioria dos acadêmicos respondeu corretamente que o tétano não é transmissível de um indivíduo para outro e ocorre em pessoas não imunes, ou seja, sem níveis adequados de anticorpos protetores (GOMERI; GAGLIANI, 2011), e foi o curso de Odontologia, mais uma vez, que apresentou menos conhecimento sobre a não transmissibilidade da doença tétano.

Entre os acadêmicos pesquisados neste estudo, nenhum acadêmico afirmou ter tido a doença durante sua vida, apesar de muitos deles não terem sido vacinados. Sabe-se que o tétano contraído de forma acidental ocorre em pessoas não vacinadas ou que receberam esquemas vacinais incompletos (GOMERI; GAGLIANI, 2011).

Quanto ao agente etiológico para a doença tétano (Tabela 2), a maioria dos alunos afirmou saber que se trata de uma bactéria, o Clostridium tetani. No ambiente, estão na maioria das vezes em sua forma de resistência, os esporos. São encontrados habitualmente no solo, em áreas rurais, na pele (íntegra), nos intestinos e fezes de animais (cavalos, bois, carneiros, porcos, galinhas etc.) e de seres humanos sem causar a doença. Quando em condições anaeróbicas (ausência de oxigênio), como ocorre em ferimentos, os esporos germinam para a forma vegetativa, onde se multiplica e produz duas exotoxinas, a tetanolisina e a tetanopasmina, que são disseminadas através do sistema circulatório (sanguíneo e linfático) (BARRAVIEIRA, 1999).

A Tabela 3 traz o conhecimento dos alunos acerca da quantidade de doses para maior efetividade da vacina antitetânica. Este dado é relevante, pois é preciso que os alunos saibam quantas doses são necessárias para que possam realizar de forma adequada todo o esquema vacinal. O Ministério da Saúde recomenda a administração de três doses no primeiro ano de vida, seguidas por doses de reforço aos 15 meses e dos 4 aos 6 anos de idade; todavia, recomenda-se dose de reforço a cada dez anos, após a última dose. Em relação à recomendação da dose de reforço, esta deve ser feita com a dupla bacteriana (dT), utilizada a partir de 7 anos de idade. Mulheres que engravidarem e tiverem uma história de reforço há mais de cinco anos devem antecipar o reforço; recomenda-se a mesma antecipação do reforço vacinal nos casos de acidentes com avaliação de risco para tétano (BRASIL, 2014).

Embora bem estabelecida a necessidade de reforço para o tétano, grande parcela de acadêmicos desconhece e, por conseguinte, deixa claro que não a realizam. A necessidade de reforço deve-se 
aos níveis protetores de anticorpos do tétano, que decrescem com a idade (MORAES, 2002).

Quanto aos sinais de manifestação da doença, a maioria dos alunos mostrou conhecimentos, havendo manifestações menos grave e mais severas. Clinicamente, o tétano acidental pode se manifestar por: dificuldade de deglutição (disfagia), hipertonia mantida dos músculos masseteres (trismo e riso sardônico) dos músculos do pescoço (rigidez de nuca), contratura muscular da região dorsal (opistótono), rigidez muscular progressiva, atingindo os músculos reto-abdominais (abdome em tábua) e o diafragma, levando à insuficiência respiratória, podendo evoluir com contraturas generalizadas. As crises de contraturas geralmente são desencadeadas por estímulos luminosos, sonoros, alterações de temperatura e as manipulações do doente (BARRAVIEIRA, 1999).

Em um estudo dos casos de tétano ocorridos na região norte de Portugal, entre 2000 e 2002, nenhum deles tinha esquema vacinal completo, $17,6 \%$ tinham esquema vacinal incompleto e $64,8 \%$ nunca tinham se vacinado (CASTRO et al., 2004). Devido aos dados alarmantes encontrados no presente estudo entre acadêmicos, e à grande exposição de profissionais de saúde aos riscos de tétano e cobertura vacinal não completa nesses indivíduos, Cavalcanti et al. (2009) defendem a obrigatoriedade da profilaxia por meio de vacinas para todo profissional de saúde, principalmente os ingressos nas universidades que ainda não iniciaram as atividade clínicas, principalmente devido ao tempo das três etapas de vacinação e os riscos em razão da inexperiência, que aumentam a susceptibilidade dos acidentes com materiais perfuro-cortantes.

A universidade possui um valor relevante na informação dos alunos. Santos et al. (2006) afirmam que as instituições de ensino têm papel primordial na prevenção e controle das doenças imunopreveníveis, pois é durante a formação acadêmica que se fundamentam conceitos e se constrói o conhecimento. Azambuja et al. (2004) dizem que o conhecimento adquirido na universidade é fundamental e tende a ser aplicado e repetido pelo trabalhador, quando inserido no mercado de trabalho. Os acadêmicos da área da saúde têm papel fundamental nas políticas públicas, por isso, devem estar cientes e, principalmente, imunizados. De acordo com o estudo de Oliveira et al. (2012), os acidentes mais registrados como causa de contato com o agente etiológico do tétano foram os instrumentos perfuro-cortantes, materiais de uso diário dos profissionais de saúde.

Assim, a educação em saúde tem o objetivo de promover a formação e/ou mudança de hábito e atitudes, sendo a universidade um bom local para se promover a saúde. Além disso, estimula a luta por melhoria da qualidade de vida, da conquista à saúde, da responsabilidade comunitária, da aquisição, apreensão, socialização de conhecimentos e a opção por um estilo de vida saudável (GOMERI; GAGLIANI, 2011). Os processos de educação continuada também devem ser estimulados, a fim de promover atualização e/ou aperfeiçoamento dos profissionais de saúde e educação, para melhorar a prática das ações assistenciais e preventivas, promovendo o autocuidado e o fornecimento de informações para a população (BRASIL, 2009).

\section{Conclusão}

O conhecimento dos acadêmicos sobre o tétano foi satisfatório, no entanto, alguns alunos não estavam vacinados ou sabiam sobre a necessidade do reforço da vacina antitetânica. Os acadêmicos de Medicina revelarem maior conhecimento sobre a vacina em relação aos alunos de Enfermagem e Odontologia, embora todos tenham apresentado riscos para doença.

\section{Referências}

AZAMBUJA, E. P.; PIRES, D. P.; VAZ, M. R. C. Prevenção e controle de infecção hospitalar: as interfaces com o processo de formação do trabalhador. Texto \& Contexto Enfermagem, v. 13, n. esp., p. 79-86, 2004.

BARNETT, V. Elements of sampling theory. Kent: Hodden and Stanghton Educational, 1982.

BARRAVIEIRA, B. Tétano: educação médica continuada em infectologia. São Paulo: EPUB, 1999. $56 \mathrm{p}$.

BRASIL. Ministério da Saúde. Secretaria de Vigilância em Saúde. Guia de vigilância epidemiológica. 7. ed. Brasília: Ministério da Saúde, 2009.

BRASIL. Ministério da Saúde. Fundação Nacional de Saúde. Manual de normas e procedimentos para vacinação. 1. Ed. Brasília: Ministério da Saúde, 2014. 
CASTRO, L.; GONÇALVES, G.; CATARINO, J. Caracterização epidemiológica dos casos declarados de tétano. Oportunidades perdidas de vacinação. Acta Medica Portuguesa, v. 17, p. 225-229, 2004.

CAVALCANTI, F. M. et al. Hepatite B: conhecimento e vacinação entre os acadêmicos da Faculdade de Odontologia de Caruaru - PE. Odontologia Clínico-Cientifica, v. 8, n. 1, p. 59-65, 2009.

FEIJÃO, A. R. et al. Tétano acidental no Estado do Ceará, entre 2002 e 2005. Revista da Sociedade Brasileira de Medicina Tropical, v. 40, n. 4, p. 426-430, 2007.

FRANÇA, G. V. Riscos ocupacionais da equipe de saúde: aspectos éticos e legais. In: CONGRESSO DA ASSOCIAÇÃO MÉDICA FLUMINENSE, 20., 1999, Niterói. Anais... Niterói, 1999.

GARCIA, L. P.; FACCHINI, L. A. Vacinação contra a hepatite $\mathrm{B}$ entre trabalhadores da atenção básica à saúde. Cadernos de Saúde Pública, v. 24, n. 5, p. 1130-1140, 2008.

GOMERI, A. M. Q.; GAGLIANI, L. H. Estudo epidemiológico do tétano acidental no Brasil. Revista Unilus Ensino e Pesquisa, v. 8, n. 15, p. 20-31, 2011.

GOUVEIA, P. A. C. et al. Tendência temporal do tétano acidental no período de 1981 a 2004 em Pernambuco com avaliação do impacto da assistência em unidade de terapia intensiva sobre a letalidade. Revista da Sociedade Brasileira de Medicina Tropical, v. 42, n. 1, p. 54-57, 2009.

MORAES, E. M.; PEDROSO, E. R. P. Tétano no Brasil: Doença do Idoso? Revista da Sociedade Brasileira de Medicina Tropical, v. 33, n. 3, p. 271-275, 2000.

MORAES, E. N. Avaliação da imunidade antitetânica em idosos de asilos e grupos de convivência de
Belo Horizonte: nível de proteção atual e análise da soroconversão da vacina dupla tipo adulto. 2002. 103 f. Tese (Doutorado) - Universidade Federal de Minas Gerais, Belo Horizonte, 2002.

OLIVEIRA, J. V. R. Análise sócio-ambiental dos casos notificados de tétano acidental no Estado de Sergipe-Brasil no período de 2001 a 2006. 2008. 51 f. Dissertação (Mestrado em Saúde e Ambiente) - Universidade Tiradentes, Aracaju, 2008.

OLIVEIRA, V. C. et al. Situação vacinal contra o tétano em trabalhadores da Empresa Municipal de Obras Públicas do Município de Divinópolis, Estado de Minas Gerais, Brasil, 2009. Epidemiologia e Serviços de Saúde, v. 21, n. 3, p. 497-504, 2012.

PINHEIRO, J.; ZEITOUNE, R. C. G. Hepatite b: Conhecimento e medidas de biossegurança e a saúde do trabalhador de enfermagem. Escola Anna Nery Revista de Enfermagem, v. 12, p. 258-264, 2008.

SANTOS, S. L. V. et al. O papel das instituições de ensino superior na prevenção das doenças imunopreveníveis. Revista Eletrônica de Enfermagem, v. 8, n. 1, p. 91-98, 2006.

SILVA-JUNIOR, M. F. et al. Conhecimento dos acadêmicos de odontologia da Ufes sobre a necessidade de imunização. Revista Brasileira de Pesquisa em Saúde, v. 15, n. 4, p. 87-94, 2013.

VERONESI, R.; FOCACCIA, R. Tratado de infectologia. 5. ed. São Paulo: Atheneu, 2015. $2320 \mathrm{p}$.

VIERTEL, I. L.; AMORIN, L.; PIAZZA, U. Tétano acidental no Estado de Santa Catarina, Brasil: aspectos epidemiológicos. Epidemiologia e Serviços de Saúde, v. 14, n. 1, p. 33-40, 2005. 